\title{
Interference Utilization Precoding in Multi-Cluster loT Networks
}

\author{
Yuanchen Wang ${ }^{1,2}$, Eng Gee Lim ${ }^{2 *}$ Xiaoping Xue ${ }^{3}$, Guangyu Zhu ${ }^{4}$, Rui Pei ${ }^{5}$ and \\ Zhongxiang $\mathrm{Wei}^{3}$
}

${ }^{1}$ Department of Electrical and Electronics Engineering, University of Liverpool, Liverpool, United Kingdom, ${ }^{2}$ School of Advanced Technology, Xi'an Jiaotong-Liverpool University, Suzhou, China, ${ }^{3}$ College of Electronic and Information, Tongji University, Shanghai, China, ${ }^{4}$ Chongqing Engineering Research Center of New Energy Storage Devices and Applications, Chongqing University of Arts and Sciences, Chongqing, China, ${ }^{5}$ College of Information Science and Technology, Donghua University, Shanghai, China

In Internet-of-Things, downlink multi-device interference has long been considered as a harmful element deteriorating system performance, and thus the principle of the classic interference-mitigation based precoding is to suppress the multi-device interference by exploiting the spatial orthogonality. In recent years, a judicious interference utilization precoding has been developed, which is capable of exploiting multi-device interference as a beneficial element for improving device's reception performance, thus reducing downlink communication latency. In this review paper, we aim to review the emerging interference utilization precoding techniques. We first briefly introduce the concept of constructive

OPEN ACCESS

Edited by:

M. L. Dennis Wong,

Heriot-Watt University, Malaysia

Reviewed by:

Tong Bai,

Beihang University, China

Nazar Emirov,

Boston College, United States

*Correspondence: Eng Gee Lim enggee.lim@xjtlu.edu.cn

Specialty section:

This article was submitted to Signal Processing Theory, a section of the journal Frontiers in Signal Processing

Received: 20 August 2021 Accepted: 19 October 2021 Published: 22 November 2021

Citation:

Wang Y, Lim EG, Xue X, Zhu G, Pei R and Wei $Z$ (2021) Interference Utilization Precoding in Multi-Cluster IOT Networks.

Front. Sig. Proc. 1:761559. doi: 10.3389/frsip.2021.761559 interference, and then we present two generic downlink interference-utilization optimizations, which utilizes the multi-device interference for enhancing system performance. Afterwards, the application of interference utilization precoding is discussed in multi-cluster scenario. Finally, some open challenges and future research topics are envisaged.

Keywords: interference utilization, multi-device interference, multi-cluster, precoding design, Internet-of-Things

\section{INTRODUCTION}

Downlink precoding has been regarded as a key technology in multi-user multiple-input and multiple-output (MIMO) communications. With the channel state information (CSI) available at the base station, the multi-user interference can be calculated prior to transmission. In this way, the interference mitigation (IM)-based precoder techniques have been extensively investigated to strictly suppress the interference. The dirty-paper coding (DPC) scheme was proposed in Costa (1983) by pre-subtracting the interference prior to transmission for achieving capacity, which however assumes infinite alphabet input and incurs high computational cost. Although the Tomlinson-Harashima precoding (THP) Sun and Lei (2013) and vector perturbation (VP) Hochwald et al. (2005) precoders aim to reduce the computational complexity over the DPC approach, they still need a sophisticated sphere-search algorithm for algorithm implementation. Hence, low-complexity linear precoders, such as zero-forcing (ZF) and minimum mean squared error (MMSE) Tse and Viswanath (2005), Peel et al. (2005), have attracted much attention in practices due to their low-complexity. On the other hand, optimization-based precoding has been a popular research topic. For example, signal-tointerference-plus-noise ratio (SINR) balancing aims to maximize the minimum SINR subject to a total power constraint (Wiesel et al., 2006); transmission power minimization problem aims to reduce the transmission power at the base station, subject to user's minimum SINR requirements (Schubert and Boche, 2004). 
The above designs treat the input as infinite Gaussian signal. Hence, they only exploit the channel correlation for the precoding design. In practice, modulation size is finite, and the input is not Gaussian signal. In this case, there is scope to jointly exploit the correlation among the channels and transmitted data, so that the multi-user interference is possible to make constructive at each receiver, termed as interference utilization (IE) precoding (Masouros and Alsusa, 2007). The concept of constructive interference (CI) has been applied for anonymous communications (Wei et al., 2021a), cognitive radio (Law and Masouros, 2018), large-scale MIMO (Amadori and Masouros, 2016, 2017b), constant envelope (Amadori and Masouros, 2017a; Liu et al., 2017), hybrid beamforming (Hegde et al., 2019), multicell coordination (Wei et al., 2020b), rate-splitting (Salem et al., 2019), physical layer security (Khandaker et al., 2019; Wei et al., 2020a; Wei and Masouros, 2020), directional modulation (Wei et al., 2021b), and integrated sensing and communication systems (Liu et al., 2018). In the following section, we briefly discuss the IE-based precoder design.

\section{IE-BASED PRECODER DESIGN}

For comparison, let us first consider a classic power minimization problem subject to per-device's signal-to-interference-plus-noise ratio (SINR) requirement. Assume that the transmitter is equipped with $N$ antennas for serving $K$ devices $(N \geq K)$. Define $\boldsymbol{w}_{i} \in \mathbb{C}^{(N \times 1)}$ as the precoder vector for the $i$-th device's intended signal $s_{i}$. Write the transmitted symbol vector $\boldsymbol{s}=\left[s_{1}, \ldots, s_{K}\right]^{\mathbf{T}} \in \mathbb{C}^{(K \times 1)}$, the signal received by the $i$-th user can be written as

$$
y_{i}=\boldsymbol{h}_{i}\left[\boldsymbol{w}_{1}, \ldots, \boldsymbol{w}_{K}\right] \boldsymbol{s}+n_{i}
$$

where $\boldsymbol{h}_{i} \in \mathbb{C}^{(1 \times N)}$ is the multiple-input and single-output (MISO) channel spanning from the transmitter to the $i$-th device, while $n_{i}$ denotes the receiver's noise, following a Gaussian distribution $\mathbb{C N}\left(0, \sigma^{2}\right)$. A generic power minimization problem can be formulated as

$$
\begin{gathered}
\mathrm{P} 1: \min _{w_{1}, \ldots, w_{K}} \sum_{i=1}^{K}\left\|w_{i}\right\|^{2}, \\
\text { s.t. }(\mathrm{C} 1): \frac{\left|h_{i} w_{i}\right|^{2}}{\sum_{j \neq i}\left|h_{i} w_{j}\right|^{2}+\sigma^{2}} \geq \Gamma_{i}, \quad \forall i \in K,
\end{gathered}
$$

where $\Gamma_{i}$ is the $i$-th device's SINR requirement. The problem P1 represents a non-convex second-order cone programming (SOCP) exercise. By defining $\boldsymbol{W}_{i}=\boldsymbol{w}_{i} \boldsymbol{w}_{i}^{H} \in \mathbb{C}^{N \times N}, \mathrm{P} 1$ can be equivalently transformed into

$$
\begin{gathered}
\text { P2: } \min _{\boldsymbol{w}_{1}, \ldots, \boldsymbol{w}_{K}} \sum_{i=1}^{K} \operatorname{tr}\left(\boldsymbol{W}_{i}\right), \\
\text { s.t. }(\mathrm{C} 1): \boldsymbol{h}_{i} \boldsymbol{W}_{i} \boldsymbol{h}_{i}^{H} \geq \Gamma_{i}\left(\sum_{j \neq i} \boldsymbol{h}_{i} \boldsymbol{W}_{j} \boldsymbol{h}_{i}^{H}\right)+\sigma^{2}, \forall i \in K, \\
\text { (C2) : } \boldsymbol{W}_{i} \geq \mathbf{0}, \forall i \in K, \\
\text { (C) }: \operatorname{rank}\left(\boldsymbol{W}_{\mathrm{i}}\right)=1, \forall i \in K,
\end{gathered}
$$

which can be readily solved as a standard convex semi-definite programming (SDP) problem after dropping constraint (C3).

Different from IM-based precoding that needs to strictly suppress interference, the IE-based precoder is able to exploit the multi-device interference as a constructive element. Multidevice interference can be achieved by exploiting geometrical interpretation shown in Figure 1. Explicitly, we first rotate the signal $y_{i}$ by the angle of $\angle s_{i}$, and then the rotated signal can be mapped onto real axis and imaginary axis respectively. As can be seen, the received signal falls into a constructive region (in Figure 1B) if and only if the trigonometry below is ensured

$$
\left(\operatorname{Re}\left(y_{i} s_{i}^{*}\right)-\sigma \sqrt{\Gamma}\right) \cdot \tan \left(\frac{\pi}{M}\right) \geq\left|\operatorname{Im}\left(y_{i} s_{i}^{*}\right)\right|, \forall k \in K,
$$

where $M$ represents constellation size. $s_{i}^{\star}$ denotes the conjugate of $s_{i}$, where $s_{i}$ is the intended symbol for the $i$-th user. In particular, $\Gamma$ physically represents the Euclidean distance in the signal constellation between the constructive region and the decision thresholds, which also directly relates to SINR performance of the received signal. The above discussion can be extended into any order M-PSK and multi-level modulations Kabir et al. (2018). Now, we are able to give the interference utilization-based power minimization precoder such as

$$
\begin{gathered}
\mathrm{P} 1: \min _{\boldsymbol{w}_{1}, \ldots, \boldsymbol{w}_{K}} \sum_{i=1}^{K}\left\|\boldsymbol{w}_{i}\right\|^{2}, \\
\text { s.t. }(\mathrm{Cl}):\left(\operatorname{Re}\left(\boldsymbol{h}_{i}\left[\boldsymbol{w}_{1}, \ldots, \boldsymbol{w}_{K}\right] \boldsymbol{s} s_{i}^{\star}\right)-\sigma \sqrt{\Gamma}\right) . \\
\tan \left(\frac{\pi}{M}\right) \geq\left|\operatorname{Im}\left(\boldsymbol{h}_{i}\left[\boldsymbol{w}_{1}, \ldots, \boldsymbol{w}_{K}\right] \boldsymbol{s} s_{i}^{\star}\right)\right|, \forall k \in K .
\end{gathered}
$$

Evidently, the precoder optimization is convex in nature, which can be solved directly. Then, we further discuss the IE-based precoder for SINR balancing optimization. When formulating SINR balancing for IE precoder, its problem formulation can be written as

$$
\begin{gathered}
\mathrm{P} 2: \min _{\boldsymbol{w}_{1}, \ldots, \boldsymbol{w}_{K}} \sum_{i=1}^{K}\left\|\boldsymbol{w}_{i}\right\|^{2}, \\
\text { s.t. }(\mathrm{C} 1):\left(\operatorname{Re}\left(\boldsymbol{h}_{i}\left[\boldsymbol{w}_{1}, \ldots, \boldsymbol{w}_{K}\right] \boldsymbol{s} \boldsymbol{s}_{i}^{*}\right)-\sigma \sqrt{\Gamma}\right) . \\
\tan \left(\frac{\pi}{M}\right) \geq\left|\operatorname{Im}\left(\boldsymbol{h}_{i}\left[\boldsymbol{w}_{1}, \ldots, \boldsymbol{w}_{K}\right] \boldsymbol{s} s_{i}^{*}\right)\right|, \forall k \in K, \\
(\mathrm{C} 2):\left\|\left[\boldsymbol{w}_{1}, \ldots, \boldsymbol{w}_{K}\right] \boldsymbol{s}\right\|^{2} \leq P_{\max },
\end{gathered}
$$

where $P_{\max }$ denotes the power budget. It has been proved in $\mathrm{Li}$ and Masouros (2018) that, the closed-form of such an IE-based precoder is given as

$$
\boldsymbol{W}=\frac{1}{K} \boldsymbol{H}^{H}\left(\boldsymbol{H} \boldsymbol{H}^{H}\right)^{-1} \operatorname{diag}(\boldsymbol{\Lambda}) \hat{\boldsymbol{s}},
$$

where $\hat{\boldsymbol{s}}$ is given as $\hat{\boldsymbol{s}}=\left[\frac{1}{S_{1}}, \frac{1}{S_{2}}, \ldots, \frac{1}{S_{k}}\right] . \boldsymbol{\Lambda}$ is an auxiliary matrix, whose value can be calculated by a low-complexity iterative algorithm in Li and Masouros (2018). It can be seen that regardless of power minimization or SINR balancing IE-based precoders, they always have linear structure and can be solved directly, without the need of calling SDP optimization.

Here, we illustrate BER performance of the IE-based precoder, compared against the $\mathrm{ZF}$ and MMSE designs as shown in 

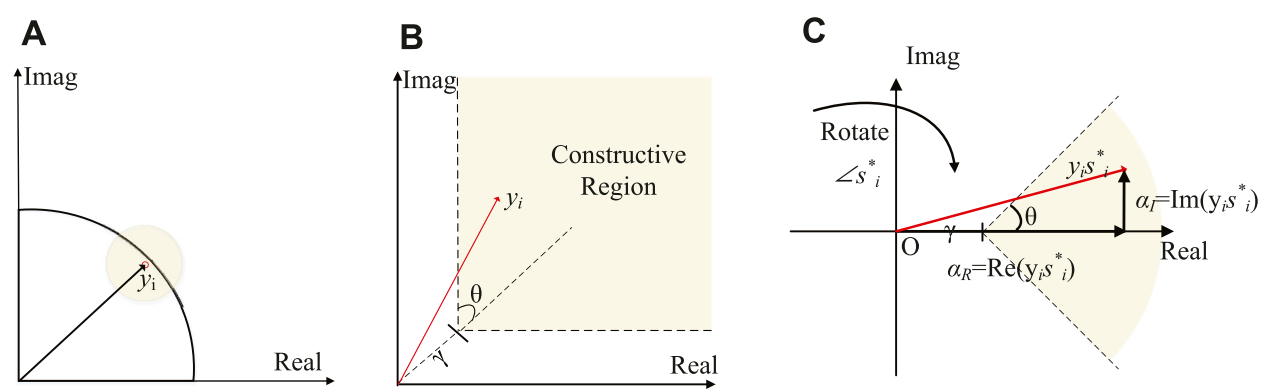

FIGURE 1 | The geometrical interpretation of IE precoding, where the intended symbol is $1+i / \sqrt{2}$ with QPSK modulation for illustration.

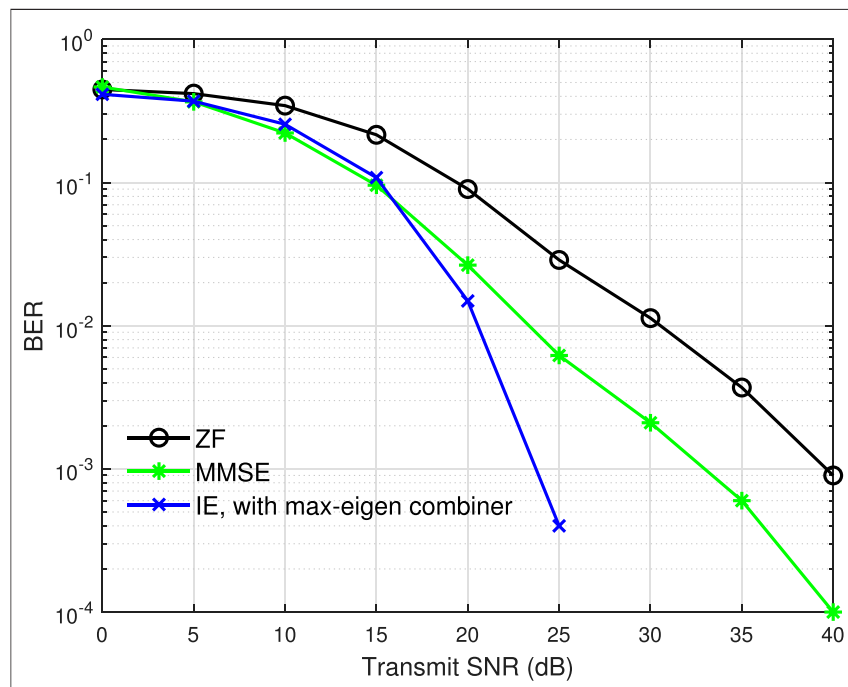

FIGURE 2 | The impact of the SNR on BER performance, where the BS is equipped with 10 transmit antennas for serving $K=10$ users. The ZF and MMSE are selected as benchmarks.

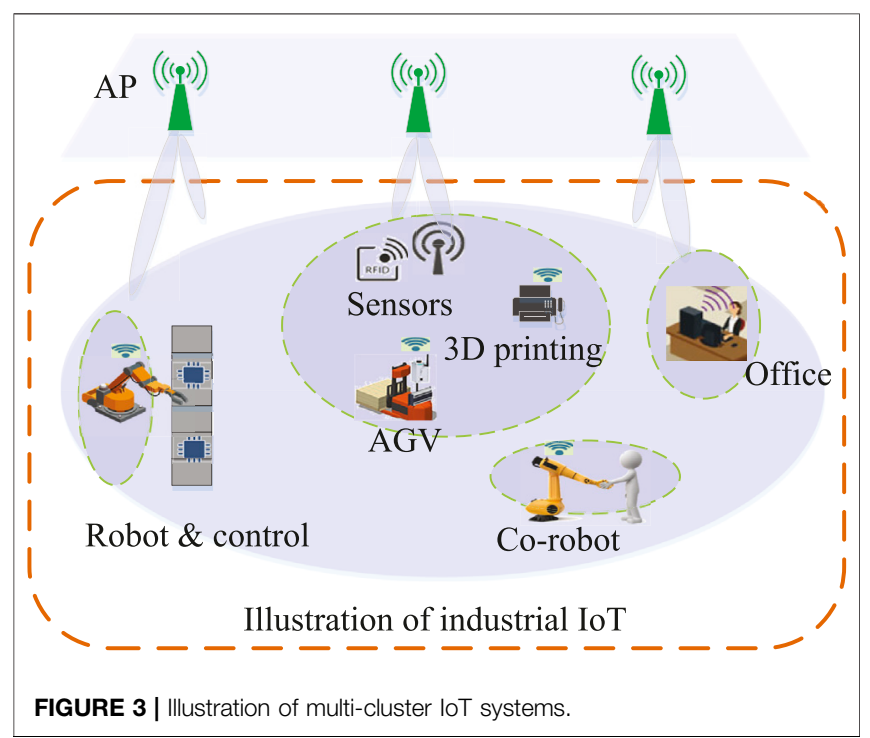

Figure 2. It is observed that as the SNR increases, the BER performance of the IE-based precoder shows rapid improvement. Furthermore, the performance of the IE-based precoder is always superior to the conventional ZF, and outperforms the MMSE at moderate/high SNR regions, which is in line with the analysis of this section.

\section{INTERFERENCE MITIGATION BASED PREOCODER IN MULTI-CLUSTER IOT NETWORKS}

In multi-cluster IoT systems shown in Figure 3, the APs are connected with high-speed optical fiber for joint signal processing. Generally, there are two different coordination mechanisms, i.e., partially-coordinated IE and fullycoordinated IE -based precoder designs. By the former design, the APs only share CSI with others for intercluster interference suppression. Since transmission data is not shared among the APs, each AP only serves its associated users, and at the same time suppresses inter-cluster interference. Assume there are $M$ APs for corporation. Define $y_{i m}$ and $n_{i m}$ as the received signal and noise at the $i$-th device belonging to the $m$-th cluster. $h_{i m} \in \mathbb{C}^{(1 \times N)}$ is the MISO channel spanning from the $m$-th AP to the $i$-th device. $\boldsymbol{W}_{\boldsymbol{m}}$ and $\boldsymbol{s}_{\boldsymbol{m}}$ denote the precoder matrix and transmitted symbol vector at the $m$-th AP, respectively. The received signal can be calculated as

$$
y_{i m}=\boldsymbol{h}_{i m} \boldsymbol{W}_{m} \boldsymbol{s}_{m}+\sum_{m^{\prime} \neq m} \boldsymbol{h}_{i m}, \boldsymbol{W}_{\boldsymbol{m}^{\prime}} \boldsymbol{s}_{\boldsymbol{m}^{\prime}}+n_{i m} .
$$

When formulating the optimization for the partially-coordinated IE precoder, the CI constraint is rewritten as

$$
\left(\operatorname{Re}\left(y_{i m} s_{i m}^{*}\right)-\sqrt{\left(\sigma^{2}+\Delta_{i m}\right) \Gamma}\right) \cdot \tan \left(\frac{\pi}{M}\right) \geq\left|\operatorname{Im}\left(y_{i m} s_{i m}^{*}\right)\right|, \forall k \in K .
$$

In particular, the term $\Delta_{i m}$ represents the inter-cluster interference at the $i$-th user, which needs to be carefully suppressed. The IE-based power minimization problem is reformulated as 


$$
\begin{gathered}
\text { P3: } \min _{W_{1}, \ldots, W_{M}} \sum_{m=1}^{M}\left\|\boldsymbol{W}_{m}\right\|^{2}, \\
\text { s.t. }(\mathrm{C} 1):\left(\operatorname{Re}\left(y_{i m} s_{i m}^{*}\right)-\left(\sigma^{2}+\Delta_{i m}\right) \Gamma\right) . \\
\tan \left(\frac{\pi}{M}\right) \geq\left|\operatorname{Im}\left(y_{i m} \boldsymbol{s}_{i m}^{*} s_{i}^{*}\right)\right|, \forall k \in K, \\
\text { (C2): } \Delta_{i m} \geq \sum_{m^{\prime} \neq m}\left\|\boldsymbol{h}_{i m}, \boldsymbol{W}_{\boldsymbol{m}} \boldsymbol{s}_{m}\right\|^{2} .
\end{gathered}
$$

In a similar vein, the IE-based SINR balancing precoder in multicluster scenario can be formulated as

$$
\begin{gathered}
\text { P4: } \max _{w_{1}, \ldots, w_{K}} \Gamma, \\
\text { s.t. }(\mathrm{C} 1):\left(\operatorname{Re}\left(y_{i m} s_{i m}^{*}\right)-\left(\sigma^{2}+\Delta_{i m}\right) \Gamma\right) . \\
\tan \left(\frac{\pi}{M}\right) \geq\left|\operatorname{Im}\left(y_{i m} s_{i}^{*}\right)\right|, \forall k \in K, \\
\text { (C2) : } \Delta_{i m} \geq \sum_{m^{\prime} \neq m}\left\|\boldsymbol{h}_{i m^{\prime}}, \boldsymbol{W}_{m^{\prime}} \boldsymbol{s}_{m^{\prime}}\right\|^{2}, \forall i \text { and } m, \\
\text { (C3) : }\left\|\boldsymbol{W}_{\boldsymbol{m}} \boldsymbol{s}_{m}\right\|^{2} \leq P_{\max }, \forall m .
\end{gathered}
$$

By contrast, the fully-coordinated IE design shares both the CSI and the data to be transmitted among the APs, where the APs jointly serve the downlink users in a similar vein of distributed antenna systems. In fact, the fully-coordinated IE makes no much difference compared to the classic IE-based precoder, as the distributed APs can be seen as a virtual multiple transmission antennas.

\section{OPEN CHALLENGES AND FUTURE RESEARCH}

The topic of the IE-based precoder is still broadly open for research and could be extended in many interesting directions:

\subsection{IE-Based Precoder in High Reliability and Low Latency Applications}

Some emerging ultra-reliability and low-latency (URLLC) applications require short packet transmission, which indeed has been considered as a key technique in the 5G URLLC scenario Sharma and Wang (2019). For example, one notable observation in these applications is that the transmitting signal is control (command) type information (e.g., start/ stop, move left/right, speed up/down, and rotate/shift) or sensing information (e.g., temperature, pressure, moisture, and gas density) (Wang Y. et al., 2021). Hence, the amount of information is delivered in short packets. Evidently, the joint design of IE-based precoder, reliability, and latency may be difficult. How to utilize the concept of IE for achieving high reliability and low latency at an acceptable degree of overhead, remains an open challenge.

\subsection{IE-Based Precoder for Millimeter-Wave MIMO Systems}

The millimeter-wave (mmWave) MIMO system is a promising technology to achieve gigabit-per-second data rates for future communications, where the number of radio-frequency (RF) chains in mmWave MIMO systems can be tens-to-hundreds of antennas (Wei et al., 2015, Wei et al., 2016). In this context, the large number of RF chains has two major issues in practice, i.e., high complexity for acquiring an optimal full-digital precoder and the hybrid precoder design (Wang J. et al., 2021). Hence, the tradeoff of IE-based Precoder design between low complexity and high reliability should be considered to suit the next-generation mmWave MIMO system.

\subsection{IE-Based Precoder for Secure Communications}

The essential feature of future communications is that of supporting massive access in IoT, and therefore, the privacy and security requirements are intended to be more complicated and diversified due to the limited number of physical resources (Wang et al., 2020). For example, the public broadcast may have a low privacy requirement, while some personal information requires high confidentiality (Chen et al., 2020). A possible solution is to classify security rank and employ appropriate techniques of physical layer security (PHY) to meet the customized demand of different users. Hence, it is demanding to fundamental analysis and new metrics for designing and evaluating the overall system PHY security performance, especially under the perspective of the IE-based secure Communications (Wei et al., 2021c).

\section{CONCLUSION}

In this review paper, we have briefly introduced the concept of IEbased precoding, two generic optimizations, i.e., power minimization and SINR balancing optimizations, are formulated. Then, we have examined the IE-based precoder design in multicluster IoT scenario. Furthermore, open challenges related to emerging applications are present, where the gap between theory and implementations should be bridged. In a nutshell, there are still essential works for the research of the IE-based precoder, which holds the promise of exciting research in the years to come.

\section{AUTHOR CONTRIBUTIONS}

All authors listed have made a substantial, direct, and intellectual contribution to the work and approved it for publication.

\section{FUNDING}

This work is supported by the AI University Research Centre (AIURC) through XJTLU Key Programme Special Fund (KSF-P-02) and Jiangsu Data Science and Cognitive Computational Engineering Research Centre.

\section{SUPPLEMENTARY MATERIAL}

The Supplementary Material for this article can be found online at: https://www.frontiersin.org/articles/10.3389/frsip.2021.761559/ full\#supplementary-material 


\section{REFERENCES}

Amadori, P. V., and Masouros, C. (2017a). Constant Envelope Precoding by Interference Exploitation in Phase Shift Keying-Modulated Multiuser Transmission. IEEE Trans. Wirel. Commun. 16, 538-550. doi:10.1109/ TWC.2016.2626279

Amadori, P. V., and Masouros, C. (2016). Interference-Driven Antenna Selection for Massive Multiuser MIMO. IEEE Trans. Veh. Technol. 65, 5944-5958. doi:10.1109/TVT.2015.2477457

Amadori, P. V., and Masouros, C. (2017b). Large Scale Antenna Selection and Precoding for Interference Exploitation. IEEE Trans. Commun. 65, 4529-4542. doi:10.1109/TCOMM.2017.2720733

Chen, L., Li, J., and Zhang, Y. (2020). Anonymous Certificate-Based Broadcast Encryption with Personalized Messages. IEEE Trans. Broadcast. 66, 867-881.

Costa, M. (1983). Writing on Dirty Paper. IEEE Trans. Inf. Theor. 29, 439-441. doi:10.1109/TIT.1983.1056659

Hegde, G., Masouros, C., and Pesavento, M. (2019). Interference ExploitationBased Hybrid Precoding with Robustness against Phase Errors. IEEE Trans. Wirel. Commun. 18, 3683-3696. doi:10.1109/TWC.2019.2917064

Hochwald, B., Peel, C., and Swindlehurst, A. (2005). A Vector-Perturbation Technique for Near-Capacity Multiantenna Multiuser Communication-Part II: Perturbation. IEEE Trans. Commun. 53, 537-544. doi:10.1109/ TCOMM.2004.841997

Kabir, M. T., Khandaker, M. R. A., and Masouros, C. (2018). Robust Energy Harvesting FD Transmission: Interference Suppression versus Exploitation. IEEE Commun. Lett. 22, 1866-1869. doi:10.1109/ LCOMM.2018.2848929

Khandaker, M. R. A., Masouros, C., Wong, K.-K., and Timotheou, S. (2019). Secure SWIPT by Exploiting Constructive Interference and Artificial Noise. IEEE Trans. Commun. 67, 1326-1340. doi:10.1109/TCOMM.2018.2874658

Law, K. L., and Masouros, C. (2018). Symbol Error Rate Minimization Precoding for Interference Exploitation. IEEE Trans. Commun. 66, 5718-5731. doi:10.1109/TCOMM.2018.2843784

Li, A., and Masouros, C. (2018). Interference Exploitation Precoding Made Practical: Optimal Closed-form Solutions for PSK Modulations. IEEE Trans. Wirel. Commun. 17, 7661-7676. doi:10.1109/TWC.2018.2869382

Liu, F., Masouros, C., Amadori, P. V., and Sun, H. (2017). An Efficient Manifold Algorithm for Constructive Interference Based Constant Envelope Precoding. IEEE Signal. Process. Lett. 24, 1542-1546. doi:10.1109/LSP.2017.2748230

Liu, F., Masouros, C., Li, A., Ratnarajah, T., and Zhou, J. (2018). MIMO Radar and Cellular Coexistence: A Power-Efficient Approach Enabled by Interference Exploitation. IEEE Trans. Signal. Process. 66, 3681-3695. doi:10.1109/ TSP.2018.2833813

Masouros, C., and Alsusa, E. (2007). A Novel Transmitter-Based SelectivePrecoding Technique for DS/CDMA Systems. IEEE Signal. Process. Lett. 14, 637-640. doi:10.1109/LSP.2007.896196

Peel, C. B., Hochwald, B. M., and Swindlehurst, A. L. (2005). A VectorPerturbation Technique for Near-Capacity Multiantenna Multiuser Communication-Part I: Channel Inversion and Regularization. IEEE Trans. Wireless Commun. 53, 195-202.

Salem, A., Masouros, C., and Wong, K.-K. (2019). Sum Rate and Fairness Analysis for the MU-MIMO Downlink under PSK Signalling: Interference Suppression vs Exploitation. IEEE Trans. Commun. 67, 6085-6098. doi:10.1109/ TCOMM.2019.2920645

Schubert, M., and Boche, H. (2004). Solution of the Multiuser Downlink Beamforming Problem with Individual SINR Constraints. IEEE Trans. Veh. Technol. 53, 18-28. doi:10.1109/TVT.2003.819629

Sharma, S. K., and Wang, X. (2019). Toward Massive Machine Type Communications in Ultra-dense Cellular IoT Networks: Current Issues and Machine Learning-Assisted Solutions. IEEE Commun. Surv. Tutor. 22, 426-471. doi:10.1109/COMST.2019.2916177

Sun, L., and Lei, M. (2013). Quantized CSI-Based Tomlinson-Harashima Precoding in Multiuser MIMO Systems. IEEE Trans. Wirel. Commun. 12, 1118-1126. doi:10.1109/TWC.2013.010413.120386
Tse, D., and Viswanath, P. (2005). Fundamentals of Wireless Communication. Cambridge University Press.

Wang, J., Zhang, X., Shi, X., and Song, J. (2021a). Higher Spectral Efficiency for mmWave MIMO: Enabling Techniques and Precoder Designs. IEEE Commun. Mag. 59, 116-122. doi:10.1109/MCOM.001.2000763

Wang, Y., Zhu, X., Lim, E. G., Wei, Z., and Jiang, Y. (2021b). Grant-Free Communications With Adaptive Period for IIoT: Sparsity and Correlation Based Joint Channel Estimation and Signal Detection. IEEE Internet Things J. doi:10.1109/JIOT.2021.3106546

Wang, Y., Zhu, X., Lim, E. G., Wei, Z., Liu, Y., and Jiang, Y. (2020). “Compressive Sensing Based User Activity Detection and Channel Estimation in Uplink NOMA Systems," in IEEE Wireless Communications and Networking Conference (WCNC) (Seoul, South Korea: IEEE), 1-6.

Wei, Z., Liu, F., Masouros, C., and Vincent Poor, H. (2021a). Fundamentals of Physical Layer Anonymous Communications: Sender Detection and Anonymous Precoding. IEEE Trans. Wirel. Commun. 1. doi:10.1109/ TWC.2021.3093722

Wei, Z., and Masouros, C. (2020). Device-Centric Distributed Antenna Transmission: Secure Precoding and Antenna Selection with Interference Exploitation. IEEE Internet Things J. 7, 2293-2308. doi:10.1109/ JIOT.2019.2958420

Wei, Z., Masouros, C., Liu, F., Chatzinotas, S., and Ottersten, B. (2020a). Energy- and Cost-Efficient Physical Layer Security in the Era of IoT: The Role of Interference. IEEE Commun. Mag. 58, 81-87. doi:10.1109/ MCOM.001.1900716

Wei, Z., Masouros, C., and Liu, F. (2021b). Secure Directional Modulation with Few-Bit Phase Shifters: Optimal and Iterative-closed-form Designs. IEEE Trans. Commun. 69, 486-500. doi:10.1109/ TCOMM.2020.3032459

Wei, Z., Masouros, C., Poor, H. V., Petropulu, A. P., and Hanzo, L. (2021c). Physical Layer Anonymous Precoding: The Path to Privacy-Preserving Communications.arXiv preprint arXiv:2109.08876.

Wei, Z., Masouros, C., Wong, K.-K., and Kang, X. (2020b). Multi-Cell Interference Exploitation: Enhancing the Power Efficiency in Cell Coordination. IEEE Trans. Wirel. Commun. 19, 547-562. doi:10.1109/ TWC.2019.2946818

Wei, Z., Zhu, X., Sun, S., Huang, Y., Dong, L., and Jiang, Y. (2015). Full-duplex versus Half-Duplex Amplify-And-Forward Relaying: Which Is More Energy Efficient in 60-GHz Dual-Hop Indoor Wireless Systems? IEEE J. Sel. Areas Commun. 33, 2936-2947. doi:10.1109/JSAC.2015.2481211

Wei, Z., Zhu, X., Sun, S., and Huang, Y. (2016). Energy-efficiency-oriented Cross-Layer Resource Allocation for Multiuser Full-Duplex DecodeAnd-Forward Indoor Relay Systems at $60 \mathrm{GHz}$. IEEE J. Sel. Areas Commun. 34, 3366-3379. doi:10.1109/JSAC.2016.2611982

Wiesel, A., Eldar, Y., and Shamai, S. (2006). Linear Precoding via Conic Optimization for Fixed MIMO Receivers. IEEE Trans. Signal. Process. 54, 161-176. doi:10.1109/TSP.2005.861073

Conflict of Interest: The authors declare that the research was conducted in the absence of any commercial or financial relationships that could be construed as a potential conflict of interest.

Publisher's Note: All claims expressed in this article are solely those of the authors and do not necessarily represent those of their affiliated organizations, or those of the publisher, the editors and the reviewers. Any product that may be evaluated in this article, or claim that may be made by its manufacturer, is not guaranteed or endorsed by the publisher.

Copyright $\odot 2021$ Wang, Lim, Xue, Zhu, Pei and Wei. This is an open-access article distributed under the terms of the Creative Commons Attribution License (CC BY). The use, distribution or reproduction in other forums is permitted, provided the original author(s) and the copyright owner(s) are credited and that the original publication in this journal is cited, in accordance with accepted academic practice. No use, distribution or reproduction is permitted which does not comply with these terms. 